\title{
PERIODIC SOLUTIONS FOR PERTURBED NONLINEAR DIFFERENTIAL EQUATIONS
}

\author{
T. G. PROCTOR ${ }^{1}$
}

\begin{abstract}
The existence of periodic solutions of a periodically perturbed system of nonlinear differential equations is established. The construction of such solutions is proved in a more restricted situation. These results generalize well-known results for perturbed linear differential equations. Examples are given.
\end{abstract}

1. Introduction. In this paper we investigate the existence and construction of periodic solutions of a periodically perturbed system of nonlinear differential equations. The perturbed system is studied using an integral equation introduced by Alekseev [1], [2] which is a generalization of the variation of constants integral equation. The techniques used are analogous to those used in establishing the existence of periodic solutions in perturbed linear systems [3]. Almost periodic perturbations of nonlinear systems have been studied by May [4] using a similar technique; however, our systems will not necessarily meet his requirements.

2. Existence of periodic solutions. Let $P, \delta, \sigma$ be positive numbers with $\delta<\sigma$, let $S_{\delta}$ and $S_{\sigma}$ be the closed spheres of radii $\delta$ and $\sigma$ respectively in $R^{n}$ and let $f(t, x)$ be a $C^{1}$ function from $R \times S_{\sigma}$ into $R^{n}$ with period $P$ in $t$. We make the following assumptions concerning the function $f$.

(i) $\gamma$ in $S_{\delta}$ implies the solution $x(t, \tau, \gamma)$ of the unperturbed initial value problem

$$
\dot{x}=f(t, x), \quad x(\tau)=\gamma
$$

exists for $0 \leqq t-\tau \leqq P$.

(ii) There is a nonempty set $K \subset S_{\delta}$ so the function $F$ given by

$$
F(\gamma)=\gamma-x(P, 0, \gamma)
$$

is a homeomorphism of $K$ onto $F(K)$. 1969.

Presented to the Society, November 22, 1969; received by the editors July 30

AMS Subject Classifications. Primary 3445.

Key Words and Phrases. Periodic solutions, perturbed nonlinear differential equations, Alekseev formula, variation of constants, existence of periodic solutions, construction of periodic solutions, Schauder fixed point theorem.

1 This work was supported by the National Aeronatuics and Space Administration under Research Grant NGR-41-001-016. 
(iii) $f(t, 0)=0,0 \leqq t \leqq P$.

We denote the continuous functions $k$ from $[0, P]$ into $S_{\delta}$ with $k(0)=k(P)$ by $\delta_{\delta}$. Let the perturbation function $g(t, x, \epsilon)$ be a continuous function from $R \times S_{\delta} \times\left[0, \epsilon_{0}\right]$ into $R^{n}$ and satisfy

(iv) $g$ has period $P$ in $t, g(t, x, 0) \equiv 0$.

(v) For $k$ in $S_{\delta}$ and

$$
\alpha(k, \epsilon)=\int_{0}^{P} \frac{\partial x}{\partial \gamma}(P, s, k(s)) g(s, k(s), \epsilon) d s,
$$

we assume $\alpha(k, \epsilon)$ is in $F(K)$ for $0 \leqq \epsilon \leqq \epsilon_{0}$.

Let $S$ be the Banach space of all continuous functions $k$ from $[0, P]$ into $R^{n}$ with $k(0)=k(P)$ and the supremum norm and let $T$ be an operator from $S_{\delta}$ into $S$ defined by

$$
T k(t)=x\left(t, 0, F^{-1}(\alpha(k, \epsilon))\right)+\int_{0}^{t} \frac{\partial x}{\partial \gamma}(t, s, k(s)) g(s, k(s), \epsilon) d s
$$

for $0 \leqq t \leqq P, 0 \leqq \epsilon \leqq \epsilon_{0}$.

Theorem 1. For $\epsilon$ small enough $T$ has a fixed point $y(t)$ in $s_{\delta}$. Further the periodic extension $y^{*}(t)$ of $y(t)$ is a periodic solution of the perturbed differential equation

$$
\dot{y}=f(t, y)+g(t, y, \epsilon) .
$$

Proof. The hypothesis on $f$ implies $x(t, \tau, \gamma)$ and $(\partial x / \partial \gamma)(t, \tau, \gamma)$ are continuous for $\gamma$ in $S_{\delta}$ and $0 \leqq t-\tau \leqq P$. Conditions (iii) and (iv) imply

$$
\lim _{\epsilon \rightarrow 0} x\left(t, 0, F^{-1}(\alpha(k, \epsilon))\right)=0
$$

uniformly for $0 \leqq t \leqq P$ and $k$ in $S_{\delta}$ so choose $\epsilon_{1}>0$ so that $T \delta_{\delta} \subseteq \delta_{\delta}$ for $0 \leqq \epsilon \leqq \epsilon_{1}$. It is easily checked that $T$ is continuous, $S_{\hat{o}}$ is closed and convex and $T \delta_{\delta}$ has a compact closure in $\delta_{\delta}$; therefore by the Schauder fixed point theorem there is a function $y(t)=T y(t)$. This representation shows $y(t)$ has a derivative which is given on $[0, P]$ by

$$
\begin{aligned}
\dot{y}(t)= & f(t, x(t))+g(t, y(t), \epsilon) \\
& +\int_{0}^{t} f_{x}(t, x(t, s, y(s))) \frac{\partial x}{\partial \gamma}(t, s, y(s)) g(s, y(s), \epsilon) d s
\end{aligned}
$$

where $x(t)=x\left(t, 0, F^{-1}(\alpha(y, \epsilon))\right)$. (Here the derivatives at 0 and $P$ are right and left derivatives respectively.) Also we have 


$$
\begin{aligned}
f(t, y(t))-f(t, x(t)) & \\
& =\int_{0}^{t} \frac{d}{d s} f(t, x(t, s, y(s))) d s \\
& =\int_{0}^{t} f_{x}(t, x(t, s, y(s))) \frac{\partial x}{\partial \gamma}(t, s, y(s))[\dot{y}(s)-f(s, y(s))] d s .
\end{aligned}
$$

Therefore for

$$
W(t)=\dot{y}(t)-g(t, y(t), \epsilon)-f(t, y(t)),
$$

we have

$$
W(t)=-\int_{0}^{t} f_{x}(t, x(t, s, y(s))) \frac{\partial x}{\partial \gamma}(t, s, y(s)) W(s) d s .
$$

The only continuous solution of this equation on $[0, P]$ is $W(t) \equiv 0$.

Example 1. The initial value problem $\dot{x}=x^{2}, x(\tau)=\gamma$ has solution

$$
x(t, \tau, \gamma)=\frac{\gamma}{1-\gamma(t-\tau)}, \quad-\frac{1}{2 P} \leqq \gamma \leqq \frac{1}{2 P}, \quad 0 \leqq t-\tau \leqq P .
$$

For $K=[-1 / 2 P, 0]$ the function

$$
F(\gamma)=\gamma-\frac{\gamma}{1-\gamma P}, \quad \gamma \text { in } K,
$$

is continuous and has continuous inverse

$$
F^{-1}(\alpha)=\frac{\alpha-\left(\alpha^{2}-4 \alpha / P\right)^{1 / 2}}{2}
$$

for $-1 / 6 P \leqq \alpha \leqq 0$. The requirement (v) on $g$ can be written as $k$ in $S_{1 / 2 P}$ implies

$$
-1 / 6 P \leqq \int_{0}^{P} \frac{1}{[1-k(s)(P-s)]^{2}} g(s, k(s), \epsilon) d s \leqq 0 .
$$

Theorem 1 establishes the existence of a periodic solution to a perturbed nonlinear differential equation; however, no construction is given for such a solution. In many cases it seems unrealistic to suppose that $T$ is a contraction operator since $F^{-1}$ may not be Lipschitz as in the example. The following section does provide a method to construct periodic solutions in a special situation. 
3. Construction of periodic solutions. Let $\Omega$ be a region in $R^{n}$, and let $f(t, x)$ be a $C^{1}$ function from $R \times \Omega$ into $R^{n}$ with period $P>0$ in $t$ and let $l(t), u(t)$ be continuous functions from $R$ into $\Omega$ with period $P$ with $l(t) \leqq u(t), 0 \leqq t \leqq P$ where a vector inequality $l \leqq u$ means the components $l_{i}, u_{i}$ of the vectors $l, u$ satisfy $l_{i} \leqq u_{i}, i=1,2, \cdots, n$. Let

$$
S=\left\{x \text { in } R^{n}: l(t) \leqq x \leqq u(t) \text { for some } t\right\}
$$

then for $\gamma$ in $S$ we assume

(i') the solution $x(t, \tau, \gamma)$ of the initial value problem $\dot{x}=f(t, x)$, $x(\tau)=\gamma$ exists for $0 \leqq t-\tau \leqq P, \tau$ any real number; and

(ii') the function $F$ given by $F(\gamma)=\gamma-x(P, 0, \gamma)$ is a homeomorphism of $S$ onto $F(S)$.

Let $g(t, x)$ be a continuous function from $R \times S$ into $R^{n}$ with period $P$ in $t$ and let $\delta^{*}$ be the set of continuous functions $k(t)$ from $[0, P]$ into $R^{n}$ with $k(0)=k(P)$ and $l(t) \leqq k(t) \leqq u(t)$ for all $t$. For $k$ in $\delta^{*}$ and

$$
\alpha(k)=\int_{0}^{P} \frac{\partial x}{\partial \gamma}(P, s, k(s)) g(s, k(s)) d s
$$

we assume $\alpha(k)$ is in $F(S)$. And we define an operator $T$ on $\S^{*}$ in to $\delta$ by

$$
T k(t)=x\left(t, 0, F^{-1}(\alpha(k))+\int_{0}^{t} \frac{\partial x}{\partial \gamma}(t, s, k(s)) g(s, k(s)) d s\right.
$$

for $0 \leqq t \leqq P$.

Theorem 2. If $l(t) \leqq T l(t), T u(t) \leqq u(t)$ for $0 \leqq t \leqq P$ and if $k, h$ in $\mathrm{s}^{*}$ with $k(t) \leqq h(t), 0 \leqq t \leqq P$ implies $T k(t) \leqq T h(t), 0 \leqq t \leqq P$, the sequences $\left\{T^{m} l\right\}_{m=0}^{\infty},\left\{T^{m} u\right\}_{m=0}^{\infty}$ converge uniformly to fixed points of T. If $y$ is a fixed point of $T$ then the periodic extension of $y(t)$ is a periodic solution of $\dot{y}=f(t, y)+g(t, y)$.

Proof. The sequences $\left\{T^{m} l(t)\right\}_{m=1}^{\infty}$ and $\left\{T^{m} u(t)\right\}_{m=0}^{\infty}$ are nondecreasing and nonincreasing respectively, uniformly bounded and equicontinuous. Hence they converge uniformly to limit functions which are fixed points of $T$. The proof that such a fixed point is a solution of the perturbed differential equation is identical to that given for Theorem 1 .

EXAmple 2. The initial value problem $\dot{x}=x(1-x), x(\tau)=\gamma$ has solution

$$
x(t, \tau, \gamma)=\gamma e^{t-\tau} /\left(1+\gamma\left(e^{t-\tau}-1\right)\right)
$$

for $\gamma>0,0 \leqq t-\tau \leqq P$. For $\gamma^{*}=\left(e^{P / 2}-1\right) /\left(e^{P}-1\right)$ the function

$$
F(\gamma)=\gamma-\gamma e^{P} /\left(1+\gamma\left(e^{P}-1\right)\right), \quad \gamma^{*} \leqq \gamma \leqq 1,
$$


has continuous inverse

$$
F^{-1}(\alpha)=\frac{\left(e^{P}-1\right)(1+\alpha)+\left(\left(e^{P}-1\right)^{2}(1+\alpha)^{2}+4 \alpha\left(e^{P}-1\right)\right)^{1 / 2}}{2\left(e^{P}-1\right)},
$$

$F\left(\gamma^{*}\right) \leqq \alpha \leqq 0$. Let $h(t)$ be a nonnegative function of period $P$ such that

$$
\int_{0}^{P} h(s) d s \leqq \gamma^{*}\left(e^{P / 2}-1\right),
$$

let

$$
g(t, x)=-h(t) /\left(1+x^{2}\right)
$$

and let $l(t)=0, u(t)=1,0 \leqq t \leqq P$. It is easy to verify the conditions of the theorem if $P$ is small enough.

4. Final remarks. The hypothesis ii and ii' of Theorems 1 and 2 respectively is analogous to the noncriticality requirement [3] made for unperturbed linear systems. The existence of periodic solutions to perturbed nonlinear systems corresponding to the critical case can be treated using the methods above. However, if one imposes hypotheses similar to those used in Theorem 1 the question of a solution to the resulting bifurcation equations is not easily established. This follows since we do not know the dependence of the fixed point $y(t)$ as a function of $\epsilon$. Hypotheses similar to those used in Theorem 2 are extremely hard to verify since this requires that the difference between a function $g(t, x)$ and its mean value be monotone over a class of periodic functions $x(t)$.

\section{REFERENCES}

1. V. M. Alekseev, An estimate for the perturbations of the solutions of ordinary differential equations. I, Vestnik Moskov. Univ. Ser. I Mat. Meh. 1961, no. 2, 28-36. (Russian) MR 23 \#A2596.

2. - An estimate for the perturbations of the solutions of ordinary differential equations. II, Vestnik Moskov. Univ. Ser. I Mat. Meh. 1961, no. 3, 3-10. (Russian) MR 24 \#A3367; MR 25, p. 1242.

3. J. K. Hale, Oscillations in nonlinear systems, McGraw-Hill, New York, 1963. MR 27 \#401.

4. L. E. May, Perturbation problems in fully nonlinear systems, Dissertation, North Carolina State University, Raleigh, N. C., 1969.

Clemson University 